126 FROM HERE TO THERE: WHAT ARE THE SPC NEEDS \& OUTCOMES OF PATIENTS ADMITTED FROM HOSPITAL TO HOSPICE IPU AND COULD THEIR NEEDS HAVE BEEN MET BY HOSPICE ADMISSION AT OUTSET?

FA Malik, SL Free, D Barclay. St. Wilfrid's Hospice, Eastbourne, East Sussex NHS Healthcare Trust

\subsection{6/bmjspcare-2018-ASPabstracts.153}

Introduction Visits to the Emergency Department by cancer patients are increasing but are associated with poor outcomes and capacity concerns. Current strategies aim to decrease hospital admissions and manage needs in community e.g. home/ hospice. However it is not known whether needs of patients admitted from hospital (HA) to hospice could have alternatively been met by hospice IPU admission at outset.

Methods Cross-sectional retrospective analysis of consecutive HA to Hospice IPU (14 bed unit) August 16-March 17. Data collected: Demographics and outcome data from hospital/hospice admission. Two clinicians assessed whether management could have been met in hospice IPU at outset. Data anonymised and treated confidentially. Results analysed and descriptive statistics utilised.

Results Data from 50 consecutive transfers collected. Mean age 71.3 years (SD 13.7) and $60 \%$ male. $82 \%$ had a cancer diagnosis (Lung (22\%) most common). More than half $(52 \%)$ of patients were known to SPC Services prior to hospital admission. 38\% (19/50) of patients self-referred to A and E. Half of admissions were in working hours $(52 \%$ admitted 9 am-5 pm Mon-Fri). Most common phase of illness on hospital 1 st assessment was unstable $(n=22,44 \%)$. Mean AKPS 38.9 (SD 22). Mean acute hospital inpatient length of stay was 14.7 days (SD 11.2; range $1-49$ days). In 38 cases it was assessed that hospice IPU could not have met immediate needs at outset. Reasons: needing procedure/operation/hospital specialty review 9/38, needs acute investigations 9/38; acute sepsis treatment $9 / 38$; chemotherapy related problems $3 / 38$, patient previously not known to have palliative diagnosis/unknown to hospice 8/38. The majority of HA (90\%) died during that hospice admission, $4(10 \%)$ discharged. Total length IPU stay 13.2 days (SD 16.4) but range broad (1-73 days).

Conclusions Most HA patients needed initial acute hospital admission at outset (rather than direct IPU admission). Findings will guide 24/7-service development and future planning of hospice beds.

\section{THE CHANGING LANDSCAPE OF ADVANCE CARE PLANNING: A MULTI-CENTRE STUDY COMPARING PRACTICE IN 2014 TO 2017}

Louisa Nelms, Anna Lock, Sarah Bache. Members of Specialist Palliative Care Audit and Guideline Group (SPAGG) including Birmingham St. Mary's Hospice, Heart of England Foundation Trust, John Taylor Hospice, Marie Curie Hospice West Midlands, Sandwell Hospital, Sandwell community team, Walsall Community Team

\subsection{6/bmjspcare-2018-ASPabstracts. 154}

Background Advance care planning (ACP) is an important part of holistic care for palliative patients. Previous studies suggest that engagement in ACP improves patient and family satisfaction with care at end-of-life.

Methods Ten specialist palliative care organisations across Birmingham contributed data to a retrospective study - designed and co-ordinated by the local Specialist Palliative Care Audit and Guideline Group (SPAGG) - across all settings including hospice inpatients, community and hospital. Data from 166 patients known to these services was collected using an electronic data collection form. The results were compared to a similar sample taken in 2014, and conclusions were drawn. Results This study found the number of patients with a documented resuscitation status, preferred place of care and death had increased from 2014. Specifically, $81 \%$ had a preferred place of death documented in 2017 compared to 58\% in 2014. Preferred place of death was achieved for $66 \%$ of patients in 2017 compared to $30 \%$ in 2014. In contrast, the number of patients completing a hand-held document of their wishes was $14 \%$ in 2017 which had fallen from 24\% in 2014. Also, documented information was less likely to be shared with other health professionals including ambulance and out of hours services in 2017 compared to 2014.

Conclusions Rates of discussions of resuscitation, preferred place of care and death have increased since 2014. As a consequence, an increased number of patients achieved their preferred place of death. This is likely due to increasing organisational and political focus on avoiding unwanted hospital deaths and staff training to improve skills and confidence in these areas. The decrease in the number of patients with a hand-held document and lack of data sharing suggests opportunities for SPAGG to focus on supporting consistent implementation of a hand-held tool and improved communication across the locality.

\section{VTE PROPHYLAXIS IN A SPECIALIST PALLIATIVE CARE UNIT}

Kirsty Lowe, Ryan Lawrie. NHS Grampian

10.1136/bmjspcare-2018-ASPabstracts. 155

Introduction NICE recommend considering VTE prophylaxis for palliative care patients with potentially reversible acute pathology, taking into account potential risks/benefits and the views of patients and families. In Roxburghe House Specialist Palliative Care Unit (RH), NHS Grampian, there is no formal decision-making process around VTE prophylaxis.

Aim To assess the current level of documentation of decisionmaking regarding VTE prophylaxis in patients admitted to RH. To subsequently introduce a VTE assessment proforma as part of the admission pack to prompt decision-making and documentation of VTE prophylaxis decisions.

Methods The notes of 19 inpatients in RH were reviewed. The following data was recorded: diagnosis, VTE prophylaxis on admission/at time of data collection, documentation of decision as well as potential risks/benefits from VTE prophylaxis.

Following this, a VTE assessment proforma was introduced in the admission documentation for RH. The notes of patients admitted following were subsequently analysed.

Results Initially, only 8 patients (42\%) admitted to $\mathrm{RH}$ had a documented decision regarding VTE prophylaxis. 10 patients $(53 \%)$ were receiving prophylactic or treatment dose Dalteparin. Of the remaining 9 patients (47\%), it was felt that 6 would not benefit from VTE prophylaxis (4 had active bleeding, 2 had cerebral tumours), and that 3 may benefit.

Following the implementation of the VTE prophylaxis proforma, Preliminary results show that 11 patients (100\%) had a documented decision in the medical notes regarding VTE 
prophylaxis. 2 patients (18\%) were started on prophylaxis and in 1 patient it was continued.

Conclusion The documentation of decision making regarding VTE prophylaxis in $\mathrm{RH}$ was initially poor. Following implementation of the assessment tool there has been improvement in documenting and discussion around VTE prophylaxis.

Data collection is still ongoing. The next step will be to assess if VTE prophylaxis prescriptions are reviewed, e.g. monitoring platelets, reviewing the duration and appropriateness of treatment.

\section{THE DEVELOPMENT OF AN ENHANCED NURSING HOME BEDS SERVICE PROVIDES AN ALTERNATIVE TO HOSPITALISATION FOR PEOPLE APPROACHING THE END OF THEIR LIVES}

Caroline McCluskey, Robert Smith. Derby Teaching Hospitals NHS Foundation Trust

\subsection{6/bmjspcare-2018-ASPabstracts. 156}

Background In 2013 the Palliative Medicine Department at Derby Hospitals launched an initiative within care homes with the aim of reducing unwanted hospitalisation and offering an alternative place of care for people approaching the end of their lives.

The Enhanced Beds Service consists of 10 beds within 5 Nursing Homes across a commissioning locality. They are available to patients who reach a crisis point, needing 24 hour care, where hospitalisation is undesirable. Day to day care is delivered by the Nursing Home staff, overseen by three specialist nurses who provide support and additional nursing care to patients and those important to them, as well as training and support to the Nursing Home staff.

Referrals are taken from all care providers including the hospital and community palliative care teams. Patients are assessed in their current location and, if admitted, are usually transferred within hours. A patient and carer experience survey and an audit are completed for all referrals. These measures monitor the service impact and direct improvements to the service.

Results In the last 4 years 466 patients have been transferred into an Enhanced Bed, $63 \%$ of which would have otherwise been admitted to hospital the same day - potentially saving 3500 acute bed days. Overall, 96\% of admissions avoid further hospitalisation. The experience survey reports a high level of satisfaction in the care received. Informal feedback from colleagues in health and social care is positive, with increasingly more complex patients being referred.

Recommendations The Enhanced Beds service has demonstrated measurable improvements in patient experience and outcomes. Future developments include a 'roaming bed' to increase choice of location.

\section{THE PRIDE OF WEST MIDLANDS PALLIATIVE CARE RESEARCH: WM CARES}

Hazel Coop, Mike Macfarlane, Matthew Doré, Clare Marlow, Sandra Prew, Derek Willis. WM CARES, NIHR, The Royal Wolverhampton NHS Trust, University of Chester

\subsection{6/bmjspcare-2018-ASPabstracts. 157}

Background Research is key for speciality development and higher medical training but there are well recognised barriers to participation in research within palliative care. The West Midlands Palliative Medicine Registrars recognised that lack of local structure and collaboration was contributing to trainees' difficulties in participating in research.

Methods The West Midlands Palliative Medicine Registrar group initiated a trainee-led collaborative, 'West Midlands Collaborative Actioning Research in End-of-life and Supportive Care' (WM CARES), which was launched in September 2016 with local stakeholder support. We hold monthly meetings at registrar training days, involving partners including the National Institute for Health Research (NIHR), local universities, palliative medicine consultants, speciality doctors and local providers of palliative care. We regularly update the wider palliative care community via newsletters, website and social media.

Results WM CARES has developed four research questions into working groups under consultant supervision. The WM CARES network enables larger, multi-site, high quality research which crosses the boundaries of any individual registrar's placement. To date, the group has presented six posters at national conferences and two journal articles are currently being prepared for publication. WM CARES has a mission to share local work, with collaboration being a central focus. This year the collaborative organised an inaugural conference, WM CARES PRIDE (Presentations in Research, Innovation, Development and Excellence) which showcased multidisciplinary research, audits and service developments from the region. There has been buy-in from the registrar body who recognise the opportunity to meet curriculum competences and explore research interests within a structured framework. Conclusion WM CARES is an innovative and collaborative trainee-led network conducting high-quality research and raising the profile of research. It enables and enthuses Palliative Medicine Registrars and the wider multidisciplinary team to learn about and be involved in research activities, which will ultimately improve patient care.

\section{INTEGRATED ONCOLOGY AND PALLIATIVE CARE: ANALYSIS OF A NEW SERVICE FOR CANCER PATIENTS}

Yukie Kano, Julie Nevin, Anna-Marie Stevens, Jayne Wood, Alison Kennett, Laila Kamal, Angela Halley, Joanne Droney. Royal Marsden NHS Foundation Trust

\subsection{6/bmjspcare-2018-ASPabstracts. 158}

Background There is growing evidence to support the benefits of early integrated palliative care (PC) for patients with advanced cancer. Within a tertiary referral cancer centre we started a new Integrated Symptom Control and PC service. The aim of this service is to proactively identify patients who would benefit from PC review and to offer earlier support. The aim of this study is to assess the feasibility and outcomes of this service.

Methods This pre-post design study was approved locally, and is part of a national programme to develop Integrated PC. Two tumour groups were selected due to their poor prognosis; renal cell cancer (RCC) and gynaecological cancer (GC). The study was conducted between October 2016 and September 2017. 316 patients (RCC 111/GC 205) were assessed as part of the new service. 286 of these patients (RCC 100/GC 186) were formally assessed for PC needs using the validated Integrated Palliative care Outcome Scale (IPOS). Descriptive statistical analysis was conducted. 